\title{
OPTOFLUIDIC DEVICE FOR HIGH RESOLUTION AND MULTIPARAMETRIC MEASUREMENT OF SINGLE BIOLOGICAL CELLS
}

\author{
Antoine Leblanc-Hotte ${ }^{1}$, Jean-Sébastien Delisle ${ }^{2}$, Sylvie Lesage ${ }^{2}$ and Yves-Alain Peter ${ }^{1}$ \\ ${ }^{1}$ Department of Engineering Physics, École Polytechnique de Montréal, Canada \\ ${ }^{2}$ Hospital Maisonneuve-Rosemont Research Center, Canada
}

\begin{abstract}
This paper reports a novel optofluidic device providing cellular sub-populations discrimination. We designed an optofluidic device based on an interferometric measurement able to discern cell types without fluorescent tags. The device yields a high sensitivity on the volume refractive index allowing for very small variations between cell populations to be detected. The optical sensing method for a single cell, at a fixed wavelength while the resonance peaks shifts, have proven to report various parameters linked to cellular intrinsic properties. Measurements collected with the device showed a significant difference between two analyzed cell types. To our knowledge, this is the only device that employs optical multiparametric and high-resolution measurements without fluorescent tags or dependency on shape or orientation on flowing cells.
\end{abstract}

\section{INTRODUCTION}

Flow cytometers are state-of-the-art equipments used mainly to classify cells within a given population. They evaluate granularity and size of hundreds of cells per second as well as identify key membrane proteins by fluorescence. However, they aren't able to differentiate some cellular sub-populations and have to use fluorescent tags to improve the specificity. Tagging manipulations are costly and laborious besides it only offers specificity over the presence of a specific protein, associated to a function, which doesn't always reflects a distinct population. Addressing these difficulties consist of the actual challenge for the advancement of research in this field.

Following this direction, we designed an optofluidic device based on an interferometric measurement able to discern cell types without fluorescent tags. The device yields a high sensitivity on the volume refractive index allowing for very small variations between cell populations to be detected. This lab-on-chip device has the potential to act as a tool for health specialists offering a supplementary specificity on diagnostic. To our knowledge, this is the only device that employs optical multiparametric and highresolution measurements without fluorescent tags or dependency on shape or orientation on flowing cells.

This device is the continuity of a previously reported microsystem [1] which measured refractive indices of homogenous liquids with a resolution as high as $1.7 \times 10^{-5}$ RIU (Refractive Index Unit). The latest reported work at uTAS 2012 on this particular device presented results on glass beads measurements [2]. We detailed the working principle, the fabrication process and the classification of glass beads with different sizes. Other publications on similar biosensors either use scattering measurement [3] or resonant transmission spectra of an immobilized cell in an out-of-plane cavity [4]. This paper reports the latest developments on cellular classification based on high resolution and multiparametric measurement of single biological cells.

\section{THEORY}

\section{Optical operation}

Figure 1 shows the sketched $3 \mathrm{D}$ view of the designed optofluidic device.

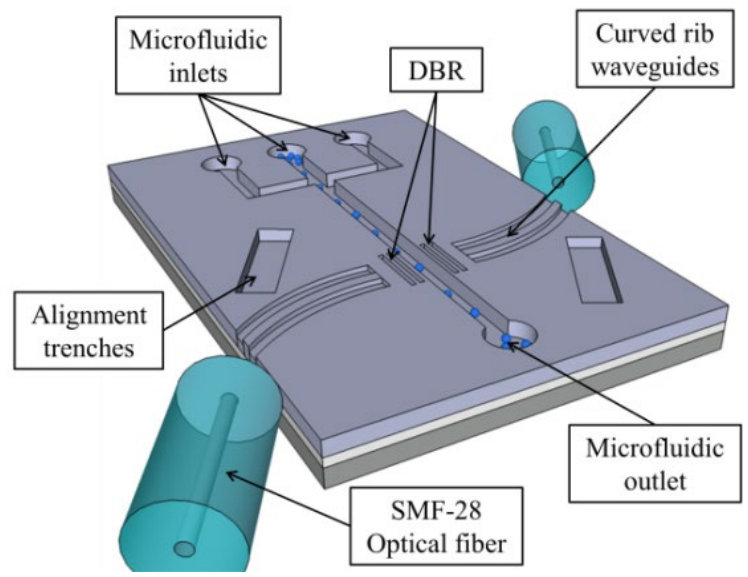

Figure 1: Sketched 3D view of the optofluidic device

Infrared light is coupled from a SMF-28 optical fiber into the integrated on-chip curved rib waveguides. These curved waveguides are used to discard the stray light from being collected in the output optical fiber. Consequently, the alignment trenches seen in the above figure are used for adjusting the angle of the optical fiber to match Snell-Descartes refraction at the interface of the curved waveguide. Also, the waveguides ends are in the shape of triangles (as shown in Figure 2) to prevent a parasitic resonant cavity between the first mirror trench and the end of the waveguide. The buried oxide layer of the SOI acts as an optical confinement structure in the z-axis because of its lower refractive index. Moreover, these rib waveguides were designed to minimize coupling loss due to mismatch of the fundamental modes shapes propagating in the optical fiber and the waveguide.

Propagating light enters a Fabry-Perot cavity consisting of two distributed Bragg mirrors separated by the width of the microfluidic channel. These distributed Bragg mirrors were designed to achieve high reflectivity as well as being easily integrated in the first etching step. Such a configuration exhibits resonance properties on the wavelength due to the optical path restriction. Resonating wavelengths will repeat at a Free Spectral Range (FSR) and their Full Width at Half Maximum (FWHM) is a function of the quality factor of the cavity, related in part to the roughness of the mirrors. To efficiently characterize this FabryPerot cavity one must use the finesse defined as:

$$
F=\frac{F S R}{F W H M}=\frac{\pi \sqrt{R}}{(1-R)}
$$

Were $\mathrm{R}$ is the mirror reflectivity. 


\section{Microfluidic operation}

Figure 2 shows a schematic view of the working principle as well as the principal defined structures from microfabrication.

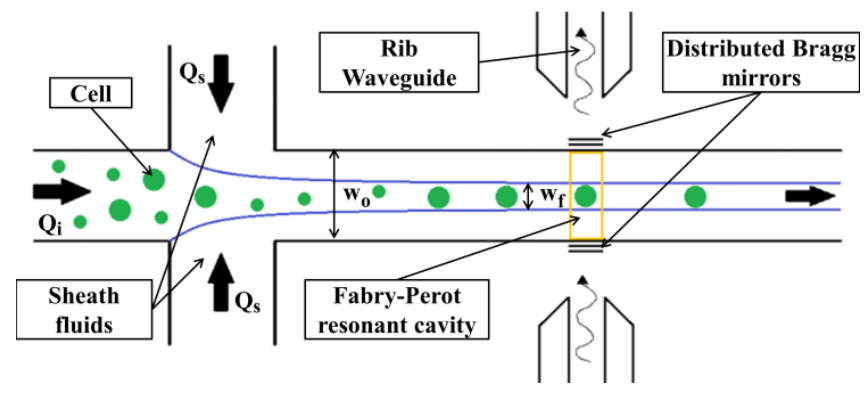

Figure 2: Schematic view of the working principle

The particular disposition of microfluidic channels enables the use of simple in-plane hydrodynamic focusing. The main governing relation is described in Equation 1, showing that only the ratio of sheath flow over inlet flow is enough to control the width of the focused flow, assuming a fixed width of the output channel.

$$
\frac{Q_{s}}{Q_{i}}=\frac{1}{2 \gamma}\left(\frac{w_{o}}{w_{f}}\right)-\frac{1}{2}
$$

Where $Q_{s}$ is the flow rate of sheath fluid, $Q_{i}$ is the flow rate of the inlet, $\mathrm{w}_{0}$ is the width of the outlet channel, $\mathrm{w}_{\mathrm{f}}$ is the width of the focused flow and $\gamma$ is a form factor varying between 1 and 1,5.

\section{Microfabrication process}

The fabrication of this device uses a 4 inches Silicon On Insulator (SOI) wafer with an $11 \mu \mathrm{m}$ thick silicon device top layer, a $2 \mu \mathrm{m}$ thick Buried Oxide layer (BOX) and a $500 \mu \mathrm{m}$ thick silicon handle layer. The complete microfabrication is achieved through a two-step process for which both steps include photolithography and Deep Reactive Ion Etching (DRIE).

Indeed, the first photolithography defines the microfluidic channels, $50 \mu \mathrm{m}$ wide in the case of the output channel, and Distributed Bragg Mirrors (DBR). These structures are then etched through the whole thickness of the top device layer. A small percentage of oxygen is used in the DRIE process to reduce the scalloping generated by the alternating plasma deposition and plasma etching of the Bosch process. The second photolithography defines the waveguides which are then etched less deeply to create the targeted rib structure. A dicing saw was employed to polish while cutting the waveguides coupling side of the chips. The specially engineered blade, a resin matrix containing diamond with a low grit size, yields a roughness on the edge of the coupling area around $25 \mathrm{~nm}$. Finally, a perforated Pyrex layer was anodically bonded on the top of the device sealing it for microfluidic operation. This top layer has ultrasonic etched holes aligned with microfluidic inlets and outlet of the device for subsequent tubes insertion and fixation with UV cured glue.

Figure 3 shows the profile of the rib waveguide and its fundamental optical mode as calculated by beam propagation method. a)

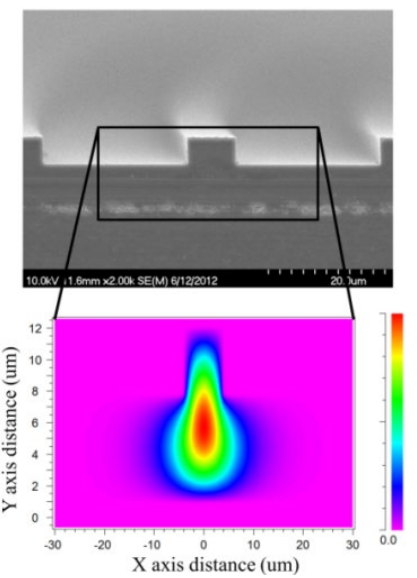

Figure $3:$ a) SEM of the microfabricated rib waveguide and b) fundamental optical mode

\section{Measurement principle}

Figure 4 shows the image of a cell at the center of the cavity.

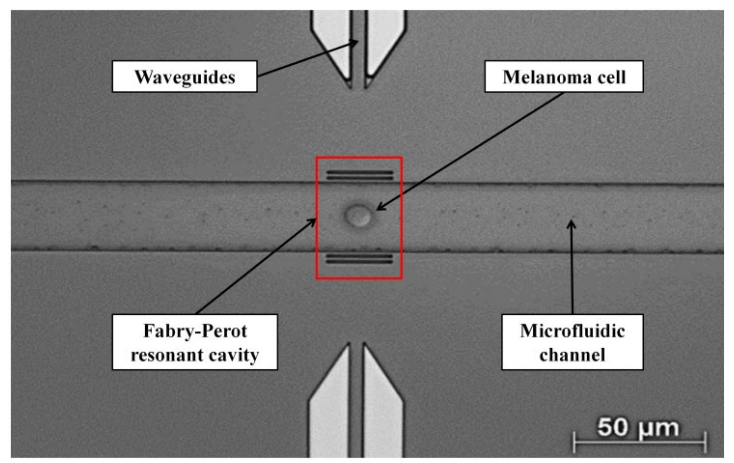

Figure 4: Cell flowing through the resonant cavity

When a cell flows through the cavity it modifies the resonance properties due to the variation of the volume refractive index. The increased optical path caused by a cell shifts the optical resonance positions towards longer wavelength as shown in Figure 5. Thus, a measurement at a fixed wavelength on the right slope of the resonance will record intensity variations. A tunable laser is used to adjust the working wavelength and an InGaAs photodetector is connected to an oscilloscope for fast acquisition of data.

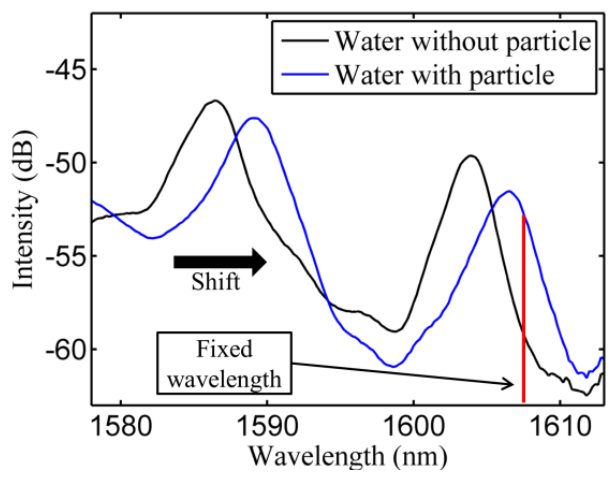

Figure 5: Position of Fabry-Perot resonance with and without a particle 
Data collected on the oscilloscope was recorded for each cell flowing in the sensing area. A trigger was set to a value slightly higher than the background noise to avoid capturing a false cellular event. This type of measurement doesn't only report the volume refractive index of the cell flowing through but also various other parameters such as maximum value, rising time, full width at half maximum (FWHM), area under the wavelength resonance, etc. as shown in Figure 6, hence the term multiparametric. These parameters are correlated to intrinsic properties of the cell, such as size, granularity, nucleus size and density, cytoplasmic volume and composition, etc. Although the exact relation between measured parameters and intrinsic properties of the cell hasn't been determined yet, discrimination of cell sub-populations can still be achieved with only two independent parameters or combination of parameters.

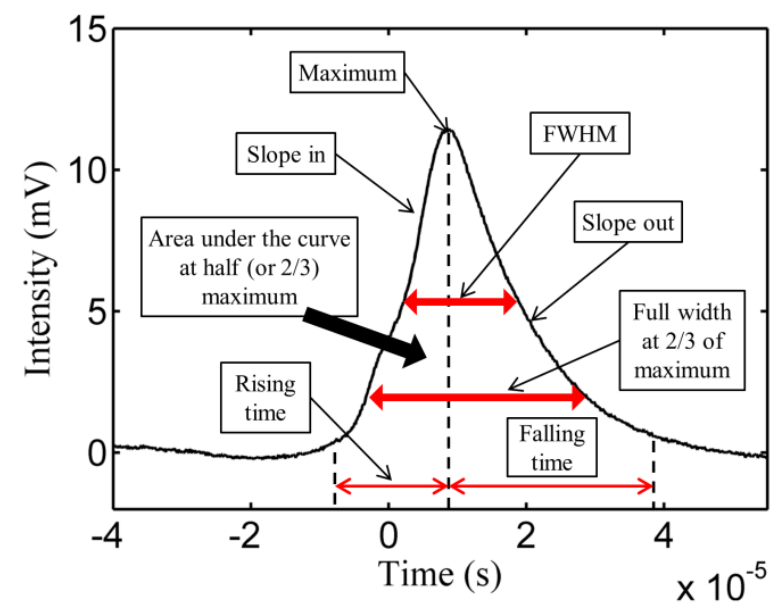

Figure 6: Parameters of a cell measurement at a fixed wavelength

\section{RESULTS}

\section{Microfluidics}

Hydrodynamic focusing was experimentally verified for different focused width. Varying the ratio of sheath flow over sample flow, as expressed in equation 1, gave a wide tunable range and control over the hydrodynamic focusing behavior.

Figure 7 shows in the upper image a low flow ratio of sheath over sample and the lower image shows a high ratio. In both cases the focused flow profile was very stable and well defined. The sample fluid was stained with trepan blue to gain optical contrast in the microscope.

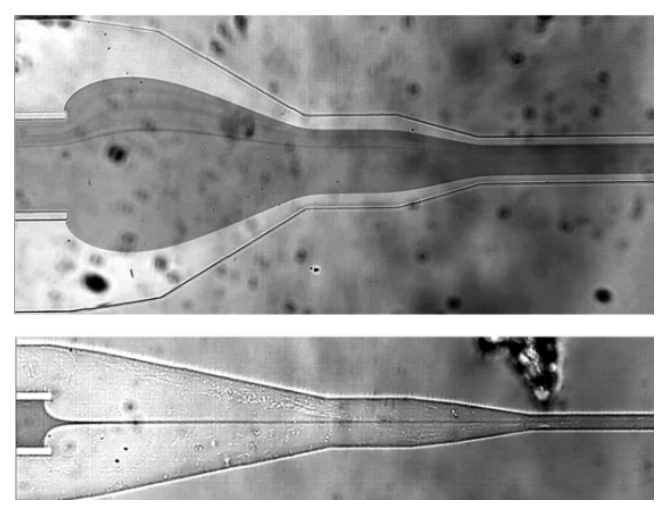

Figure 7: Hydrodynamic focusing under different conditions

\section{Optics}

Figure 8 shows the spectrum obtained from the integrated onchip Fabry-Perot.

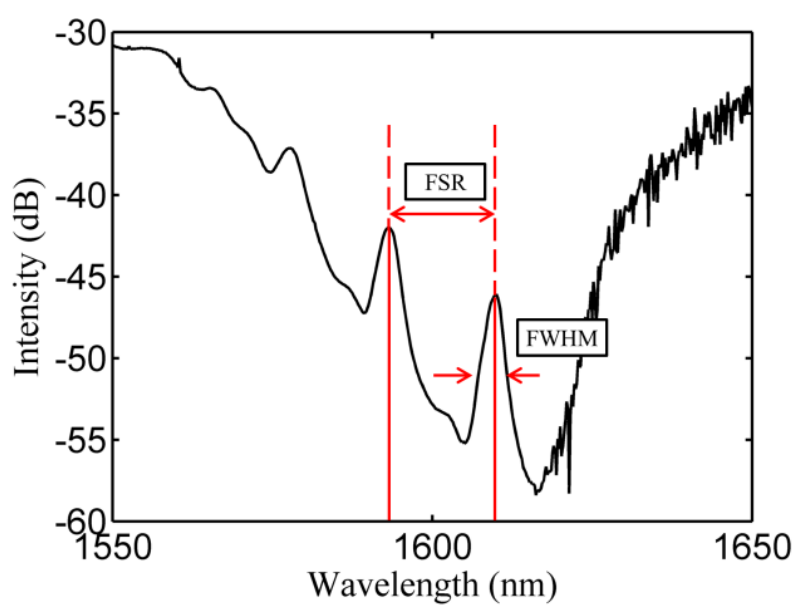

Figure 8: Typical optical spectrum from the on-chip Fabry-Perot

From the above spectrum, this device measurement revealed a FSR around $17 \mathrm{~nm}$ and a FWHM around $3.1 \mathrm{~nm}$, thus yielding a finesse around 5.5 corresponding to a reflectivity (R) of $57 \%$. Even if the finesse value is low, it is sufficient for the application described here. Indeed, assuming a sensitivity of $500 \mathrm{~nm} / \mathrm{RIU}$ and an experimental setup detection limit of $0.01 \mathrm{~nm}$ the average resolution is expected to be of $2 \times 10^{-5}$ RIU [1]. The calculated volume refractive index variation caused by a single cell $\left(\mathrm{n}_{\text {cell }}=1.36, \mathrm{~d}=10 \mathrm{um}\right)$ inside the resonant cavity filled with water $\left(\mathrm{n}_{\text {water }}=1.33, \mathrm{w}=50 \mathrm{um}\right)$ is around $4 \times 10^{-3}$ RIU. This refractive index variation is theoretically enough to be measured with our device since it corresponds to a shift around $2 \mathrm{~nm}$ of the resonance.

\section{Single cell measurements}

B16 cells and EL4 cells were cultivated following standard operating protocol and collected for analysis. B16 cells are adherent melanoma cells originating from mouse skin cancer and EL4 cells are suspended lymphoma cells originating from mouse T lymphocytes. These two immortal cell lines were chosen for a proof of concept because of their resemblance in size and granularity but still being distinguishable in a flow cytometer without fluorescent dye. Even with their resemblance, these cells have a totally different origin and role, thus a different composition and potential parameters measurable by our device.

Three different solutions were prepared for this experiment: two control solutions containing only one type of cells and a mixture in a ratio of $1: 1$. Collected measurements were statistically analyzed using a simple graphical gating program. The chosen parameters for cell classification were in this case FWHM and area under the curve at half maximum as shown in Figure 9. A comparative test was made with a Becton Dickinson FACSCalibur Flow Cytometry System without any fluorescent tags as shown in Figure 10. In both cases, each cell type was first tested alone to determine its specific region and delimitations. 


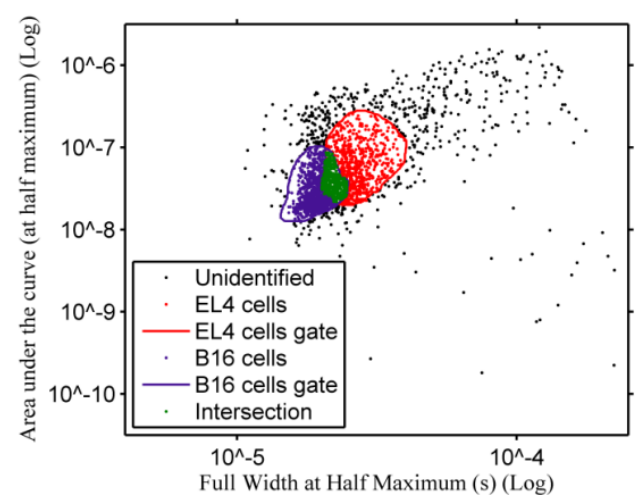

Figure 9: Typical cellular classification of the optofluidic device

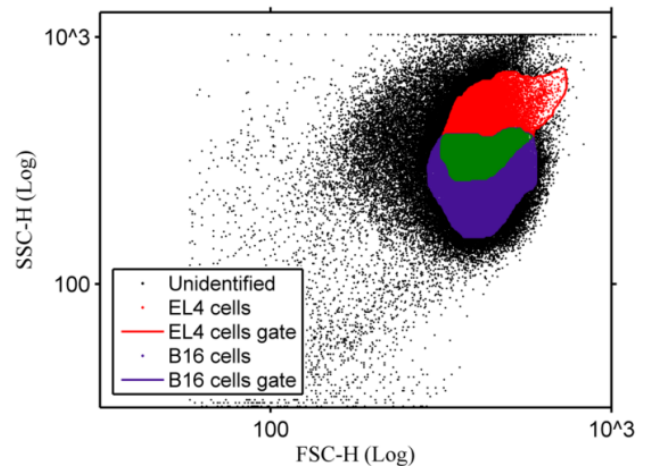

Figure 10 : Typical cellular classification of the flow cytometer

Graphically, it can be seen that for both the optofluidic device and the FACSCalibur Flow Cytometry System a similar and significant difference lies between the two cell types. Although both cell types are well identified, they still overlap each other. The magnitude of overlapping cells can be tuned by reducing the gates size of each cell type. Large gates include more cells but also render a higher overlap whereas small gates include fewer cells but a smaller overlap. The percentage of identified cells in each group is interpreted in term of sensitivity whereas the percentage of overlapping cell is interpreted as specificity. The goal is to identify the levels of sensitivity and specificity required by the experiment. Both the optofluidic device and the flow cytometer results were statistically analyzed when using large gates and small gates. Table 1 reports these statistics for the optofluidic device and Table 2 reports the statistics for the flow cytometer.

Table 1 : Identification statistics for the optofluidic device

\begin{tabular}{|c|c|c|c|c|}
\hline & \multicolumn{2}{|c|}{ Large gates } & \multicolumn{2}{c|}{ Small gates } \\
\hline & $\begin{array}{c}\text { \# of cells } \\
\text { (\% total } \\
\text { cells) }\end{array}$ & $\begin{array}{c}\% \text { on } \\
\text { identified } \\
\text { cells }\end{array}$ & $\begin{array}{c}\text { \# of cells } \\
\text { (\% total } \\
\text { cells) }\end{array}$ & $\begin{array}{c}\% \text { on } \\
\text { identified } \\
\text { cells }\end{array}$ \\
\hline Total cells & 3731 & ----- & 3731 & ---- \\
\hline $\begin{array}{c}\text { Identified } \\
\text { cells }\end{array}$ & $2683(72 \%)$ & $100 \%$ & $1312(35 \%)$ & $100 \%$ \\
\hline EL4 cells & $1667(45 \%)$ & $62 \%$ & $601(16 \%)$ & $46 \%$ \\
\hline B16 cells & $1640(44 \%)$ & $61 \%$ & $711(19 \%)$ & $54 \%$ \\
\hline $\begin{array}{c}\text { Intersection } \\
\text { cells }\end{array}$ & $624(17 \%)$ & $23 \%$ & 0 & $0 \%$ \\
\hline
\end{tabular}

Table 2 : Identification statistics for the flow cytometer

\begin{tabular}{|c|c|c|c|c|}
\hline & \multicolumn{2}{|c|}{ Large gates } & \multicolumn{2}{c|}{ Small gates } \\
\hline & $\begin{array}{c}\text { \# of cells } \\
(\% \text { total cells) }\end{array}$ & $\begin{array}{c}\% \\
\text { identified } \\
\text { cells }\end{array}$ & $\begin{array}{c}\text { \# of cells } \\
(\% \text { total cells) })\end{array}$ & $\begin{array}{c}\% \\
\text { identified } \\
\text { cells }\end{array}$ \\
\hline Total cells & 126195 & ----- & 126195 & ---- \\
\hline $\begin{array}{c}\text { Identified } \\
\text { cells }\end{array}$ & $95923(76 \%)$ & $100 \%$ & $53236(42 \%)$ & $100 \%$ \\
\hline EL4 cells & $56498(45 \%)$ & $59 \%$ & $22599(18 \%)$ & $42 \%$ \\
\hline B16 cells & $71222(56 \%)$ & $74 \%$ & $33730(27 \%)$ & $63 \%$ \\
\hline $\begin{array}{c}\text { Intersection } \\
\text { cells }\end{array}$ & $31797(25 \%)$ & $33 \%$ & $3093(2 \%)$ & $6 \%$ \\
\hline
\end{tabular}

For the optofluidic device, these values show that large gates identified $72 \%$ of total cells for which $23 \%$ of cells were overlapping thus couldn't be associated to a group. For small gates, only $35 \%$ of total cells are identified but there is no overlap in this case. On the other hand, the flow cytometer statistics with large gates show that $76 \%$ of total cells are identified for which $33 \%$ are overlapping. For small gates, only $42 \%$ of total cells are identified but the intersection cells percentage is at a lower value of $2 \%$. Moreover, the optofluidic device reports a cell type ratio close to $1: 1$ for the two gates whereas the flow cytometer reports a ratio a little further from this theoretical value.

\section{CONCLUSIONS}

In conclusion we have reported a microfabricated optofluidic device based on a Fabry-Perot resonant cavity able to discriminate cell populations. Cells are flowing through an optical sensing cavity by hydrodynamic focusing. Melanoma and lymphoma cells were analyzed with the optofluidic device and a significant difference between the two groups was found. Compared to a commercial flow cytometer, the optofluidic device gave similar results thus validating the proof of concept of our device.

\section{ACKNOWLEDGEMENTS}

This work is supported by the NSERC-CREATE ISS Training Program and travel support has been generously provided by the Transducer Research Foundation.

\section{REFERENCES}

[1] R. St-Gelais, J. Masson and Y.-A. Peter, "All-Silicon Integrated Fabry-Pérot Cavity for Volume Refractive Index Measurement in Microfluidic Systems", Applied physics letters, 94, 24 (2009).

[2] A. Leblanc-Hotte, R. St-Gelais and Y.-A. Peter, "Optofluidic Device for High Resolution Volume Refractive Index Measurement of Single Cell", International Conference on Miniaturized Systems for Chemistry and Life Sciences, Okinawa, Japan (2012), pp. 1330-1332.

[3] M. Rosenauer, W. Buchegger, I. Finoulst, P. Verhaert and M. Vellekoop, "Minituarized Flow Cytometer with 3D Hydrodynamic Particle Focusing and Integrated Optical Elements Applying Silicon Photodiodes", Microfluidics and Nanofluidics, 10 (2011), pp. 761-771.

[4] W. Wang, D.W. Kisker, D.H. Thamm, H. Shao and K. Lear, "Optofluidic Intracavity Spectroscopy of Canine Hemangiosarcoma", IEEE Transactions on Biomedical Engineering, 58, 4 (2011), pp. 853-860.

\section{CONTACT}

*Y.-A. Peter, yves-alain.peter@polymtl.ca 